\title{
Osteotomía en cuña sustractiva supracondílea para el tratamiento del codo valgo del adulto
}

\author{
Gerardo Gallucci,, Gonzalo Altube,," Jorge G. Boretto," Agustín Donndorff," Ezequiel E. Zaidenberg," \\ Ignacio Rellán," Pablo De Carli* \\ "Sector de Cirugía de Mano y Miembro Superior, Instituto de Ortopedia y Traumatología "Prof. Dr. Carlos E. Ottolenghi”, \\ Hospital Italiano de Buenos Aires, Ciudad Autónoma de Buenos Aires, Argentina \\ **Servicio de Ortopedia y Traumatología, Hospital "Enrique Vera Barros”, La Rioja, Argentina
}

\section{RESUMEN}

Objetivo: Comunicar los resultados de una serie de adultos con consolidaciones en valgo del codo tratados con una osteotomía en cuña sustractiva monoplanar. Materiales y Métodos: Se incluyeron 5 pacientes. Se describe la técnica quirúrgica que consistió en un abordaje posterior paratricipital, resección en cuña sustractiva y transposición anterior del nervio cubital. El seguimiento promedio fue de 17 meses. Resultados: Se trató a 4 hombres y una mujer, con una edad promedio de 27 años. La movilidad preoperatoria promedio fue de $138-7^{\circ}$ y la posoperatoria, de $138-6^{\circ}$, el puntaje de dolor en la EAV fue de 4 y 1 , el MEPS de 71 y 97 , y el DASH de 26 y 8 , respectivamente. La evaluación radiográfica preoperatoria arrojó un valgo promedio de $30^{\circ}$ con un valgo contralateral de $11^{\circ}$. La corrección radiográfica demostró un valgo de $13^{\circ}$. Se obtuvo una corrección promedio de $2^{\circ}$ menos que del otro lado. Todas las osteotomías consolidaron, y la medición de la prominencia medial fue, en promedio, un $32 \%$ mayor que en el preoperatorio. Según la escala de Oppenheim, el resultado fue excelente en 4 pacientes y bueno en uno. La satisfacción personal fue, en promedio, de 8,6. Conclusiones: La osteotomía en cuña sustractiva para tratar un codo valgo es una buena opción terapéutica, con recuperación de valores angulares comparables con el lado contralateral, y alta tasa de satisfacción de los pacientes. Como es una técnica menos compleja que las osteotomías multiplanares, es nuestra elección ante una consolidación viciosa en valgo del codo del adulto.

Palabras clave: Codo valgo; osteotomía; cuña sustractiva; consolidación viciosa; fractura supracondílea de codo.

Nivel de Evidencia: IV

\section{Supracondylar Subtraction Wedge Osteotomy for the Treatment of Adult Cubitus Valgus}

\begin{abstract}
Objective: To report the results of a series of adult patients with a valgus malunion of the elbow treated with a supracondylar subtractive monoplanar wedge osteotomy, Materials and Methods: 5 patients were included. The surgical technique consisted of a posterior paratricipital approach, with resection of a subtractive wedge and the anterior transposition of the ulnar nerve. The average follow-up was 17 months. Results: 4 patients were men and 1 woman with an average age of 27 years. The preoperative range of motion was $138^{\circ}-7^{\circ}$ and the postoperative range of motion was $138-6^{\circ}$. Pain according to VAS was 4 and 1 , MEPS was 71 and 97, and DASH was 26 and 8, respectively. The preoperative radiological evaluation showed an average valgus of $30^{\circ}$ with a contralateral valgus of $11^{\circ}$. The final valgus obtained was $13^{\circ}$. The final correction was, on average, $2^{\circ}$ less than the contralateral side. All osteotomies healed and the medial prominence was on average $32 \%$, more than before surgery. According to Oppenheim scale, the results were excellent in 4 patients and good in 1. Personal satisfaction was, on average, 8.6. Conclusions: Supracondylar subtractive wedge osteotomy is a good option for the treatment of adult cubitus valgus with a recovery of angular values similar to the contralateral side and a high satisfaction rate. As it is a simpler technique, compared to the multiplanar osteotomies, it is our treatment of choice for adult cubitus valgus.
\end{abstract}

Keywords: Cubitus valgus; osteotomy; subtractive wedge; malunion; supracondylar humeral fracture.

Level of Evidence: IV 


\section{INTRODUCCIÓN}

Las deformidades angulares del codo son complicaciones frecuentes de las fracturas supracondíleas en niños. Estas alteran la orientación articular de manera mono o multiplanar. ${ }^{1}$ El codo varo es la complicación más frecuente, tiene una incidencia promedio del 30\% (rango 0-60\%). ${ }^{2-4}$ Por el contrario, el codo valgo, producido por la seudoartrosis de cóndilo humeral lateral o el cierre fisario prematuro, es infrecuente. ${ }^{5}$

Esta deformidad en valgo raramente compromete la función del codo, se presenta como una alteración estética, en muchos casos, pasa inadvertida, porque supone un incremento de la posición fisiológica del codo. Sin embargo, en casos complejos, puede haber inestabilidad medial e irritación del nervio cubital inducida por su estiramiento. ${ }^{3}$

Existen distintos tipos de osteotomías para el tratamiento del cúbito valgo. ${ }^{6-8}$ Sin embargo, no hay consenso sobre cuál logra el mejor resultado final. Por lo tanto, la selección de la técnica quirúrgica depende de la preferencia del cirujano.

La osteotomía monoplanar es un procedimiento controvertido, porque no permite una corrección multiplanar que, a menudo, acompaña a la deformidad en valgo.

El objetivo de este estudio es comunicar los resultados de una serie de pacientes adultos con consolidaciones en valgo del codo tratados con una osteotomía varizante en cuña sustractiva monoplanar.

\section{MATERIALES Y MÉTODOS}

Se diseñó un estudio retrospectivo observacional que incluyó a todos los pacientes sometidos a una osteotomía correctiva de codo para corregir una deformidad, en nuestra institución, entre 2007 y 2019. Se identificó a 10 pacientes. Los criterios de inclusión fueron: pacientes $>18$ años (esqueléticamente maduros), con consolidaciones viciosas de la paleta humeral con desviación en valgo patológico $\left(>20^{\circ}\right),{ }^{7}$ tratados mediante una osteotomía varizante en cuña sustractiva medial, con estudios radiológicos preoperatorios y posoperatorios completos y un seguimiento $>1$ año.

El grupo final quedó compuesto por cinco pacientes. Los cinco restantes fueron excluidos, porque tenían consolidaciones en varo ( 3 casos) y eran $<18$ años $(2$ casos).

Todas las evaluaciones preoperatorias y posoperatorias estuvieron a cargo de uno de los autores (GG).

La evaluación radiográfica se realizó en las proyecciones regulares anteroposterior y de perfil de codo de ambas extremidades superiores, y se analizaron en forma comparativa.

Para medir la deformidad, se empleó el ángulo húmero-codo-muñeca ${ }^{8}$ en la proyección anteroposterior con el codo extendido y en máxima supinación trazando dos líneas transversales perpendiculares al eje humeral (una proximal y una distal) y dos líneas perpendiculares al eje del antebrazo (una proximal y otra distal). Luego se trazó una línea perpendicular al punto medio de las líneas humerales y otra conectando el punto medio de las líneas del antebrazo. La intersección entre estas dos líneas conforma el ángulo húmero-codo-muñeca. El ángulo de corrección necesario fue determinado utilizando el lado contralateral sano como parámetro guía.

La prominencia medial se midió con la escala de Wohn' (Figura 1).

Cuatro pacientes tenían antecedentes de una fractura supracondílea en la infancia. Uno (caso 3) había sufrido una luxación posterolateral del codo a los 11 años y, tres años antes de nuestra cirugía, sufrió un nuevo trauma con luxación. A partir de ese momento, presentó múltiples episodios de subluxación.

Dos pacientes presentaban una seudoartrosis del cóndilo humeral por una fractura tipo 2 de la clasificación de Milch. ${ }^{10}$ Dos pacientes tenían parestesias en el territorio cubital y uno (caso 1) había sido operado con una transferencia tendinosa por una parálisis cubital y transposición anterior del nervio.

Todos fueron operados por el mismo cirujano (GG).

\section{Técnica quirúrgica}

Se colocó a los pacientes en decúbito ventral con el brazo apoyado sobre una mesa quirúrgica radiolúcida. Se realizó un abordaje posterior y se identificó el nervio cubital que fue transpuesto anteriormente y en el plano subcutáneo en cuatro de los cinco casos. El paciente restante era el que ya había sido operado. En la cirugía de la osteotomía, solo se identificó el nervio, no se realizó ningún gesto quirúrgico sobre él.

Se utilizó el abordaje paratricipital en todos los casos. Antes se había medido y confeccionado la cuña por resecar en una plantilla plástica y, con ella, se realizó la osteotomía. Antes se había creado un orificio en el vértice de la cuña a $2 \mathrm{~mm}$ de la cortical externa del húmero para intentar que la osteotomía sea incompleta y facilitar la compresión. Se fijó transitoriamente con dos clavos de Steinmann de $2 \mathrm{~mm}$ y luego se procedió a la osteosíntesis definitiva con dos placas bloqueadas. 


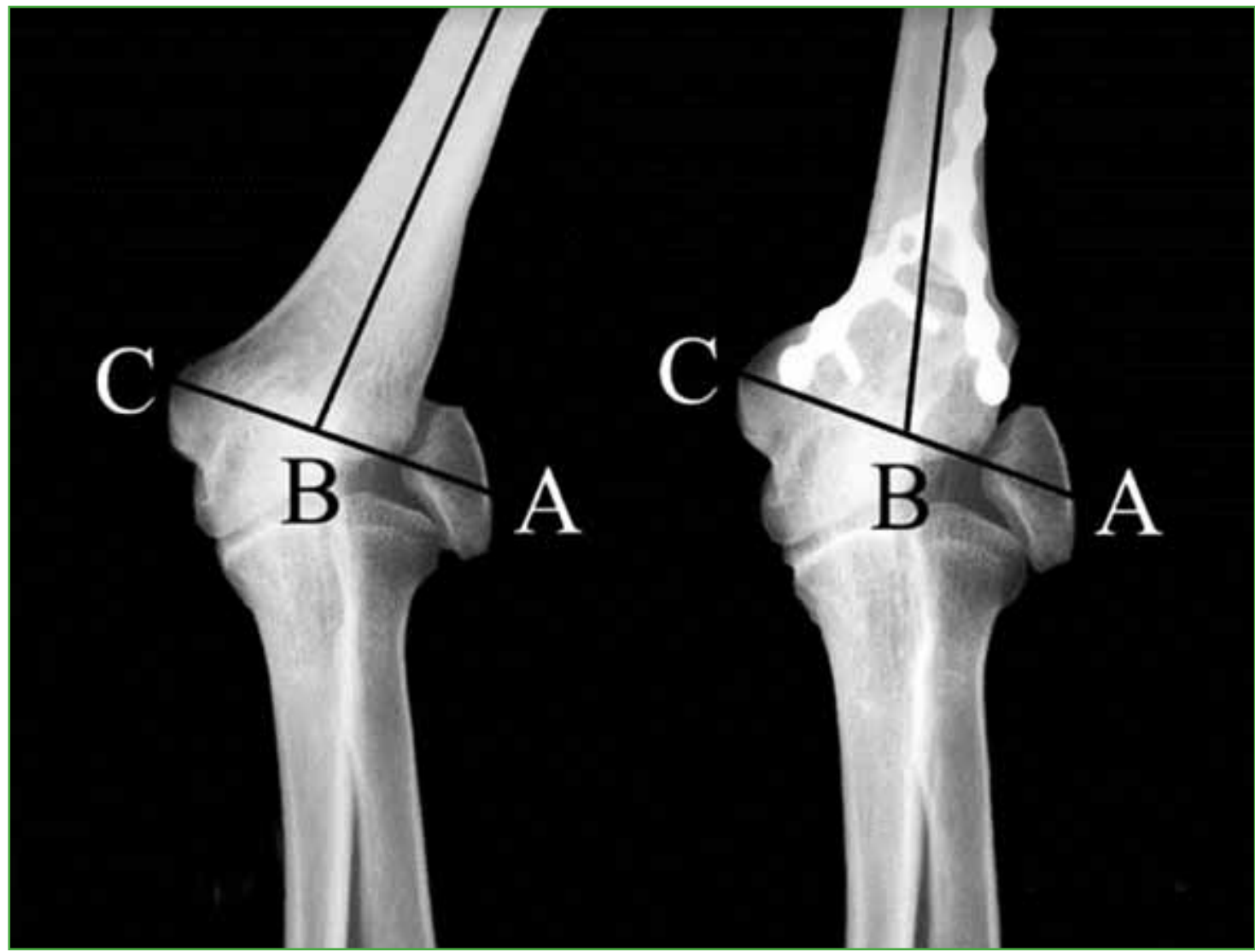

Figura 1. Medición del índice de prominencia medial $(\%)=(\mathrm{CB}-\mathrm{BA}) / \mathrm{CA} \times 100$.

Uno de los pacientes tenía inestabilidad posterolateral de codo y, en el mismo momento, se prolongó el abordaje a distal y, a través del intervalo entre el ancóneo y el cubital posterior, se accedió a la cápsula articular y se llevó a cabo la reconstrucción del ligamento lateral con una lonja de tríceps.

El cierre se hizo con un drenaje en todos los casos y la inmovilización posoperatoria, con una valva de yeso braquipalmar a $90^{\circ}$ durante una semana. En el caso de la reconstrucción ligamentaria, la inmovilización se prolongó por seis semanas.

La evaluación clínica final consistió en un examen objetivo midiendo el rango de movilidad con un goniómetro y uno subjetivo mediante los puntajes DASH (Disabilities of the Arm, Shoulder and Elbow), ${ }^{11}$ MEPS (Mayo Elbow Performance Score $)^{12}$ y la escala analógica visual (EAV) para el dolor en actividad y la satisfacción del paciente.

Para analizar los resultados clínicos de la corrección de la deformidad, se empleó la escala de Oppenheim, ${ }^{13}$ basándonos en la medición del ángulo húmero-codo-muñeca, el rango de movilidad activo y la presencia de complicaciones. El resultado se considera excelente si la corrección del ángulo húmero-codo-muñeca tiene una diferencia $<5^{\circ}$ respecto del lado contralateral, la pérdida del rango de movilidad es $<5^{\circ}$ y no hay complicaciones; bueno si la diferencia del ángulo húmero-codo-muñeca es $6^{\circ}$ a $10^{\circ}$ y la pérdida del rango de movilidad es $<6^{\circ}-10^{\circ}$ respecto del codo contralateral, acompañado de leve deformidad, y pobre si la diferencia es $>10^{\circ}$ y la pérdida del rango de movilidad es $>10^{\circ}$ en relación con el codo contralateral.

El seguimiento promedio fue de 19 meses (rango 12-37). 


\section{RESULTADOS}

El grupo estaba formado por cuatro hombres y una mujer, con una edad promedio de 27 años (rango 18-42) (Tabla 1). El lado derecho estaba afectado en cuatro casos y el miembro dominante, en otros cuatro. La movilidad preoperatoria promedio fue de $138-7^{\circ}$ y la posoperatoria, de $138-6^{\circ}$, el puntaje de dolor según la EAV fue de 4 y 1, el puntaje MEPS, de 71 y 97, y el DASH de 26 y 8, respectivamente (Tabla 2).

En dos pacientes con parestesias cubitales, los síntomas reaparecieron.

Tabla 1. Datos demográficos

\begin{tabular}{|c|c|c|c|c|c|c|c|c|c|c|c|c|}
\hline \multirow[t]{2}{*}{ Paciente } & \multirow[t]{2}{*}{ Edad } & \multirow[t]{2}{*}{ Sexo } & \multirow{2}{*}{$\begin{array}{c}\text { Lesiones } \\
\text { asociadas }\end{array}$} & \multirow{2}{*}{$\begin{array}{c}\text { Valgo } \\
\left({ }^{\circ}\right)\end{array}$} & \multirow{2}{*}{$\begin{array}{c}\text { Valgo } \\
\text { contralateral } \\
\left(^{\circ}\right)\end{array}$} & \multirow{2}{*}{$\begin{array}{c}\text { Cuña } \\
\text { resecada } \\
\left({ }^{\circ}\right)\end{array}$} & \multirow{2}{*}{$\begin{array}{l}\text { Valgo } \\
\text { final } \\
\left({ }^{\circ}\right)\end{array}$} & \multirow{2}{*}{$\begin{array}{l}\text { Correc- } \\
\text { ción } \\
\left({ }^{\circ}\right)\end{array}$} & \multicolumn{3}{|c|}{ Prominencia medial } & \multirow{2}{*}{$\begin{array}{l}\text { Seguimiento } \\
\text { (meses) }\end{array}$} \\
\hline & & & & & & & & & Preop. & Posop. & $\%$ & \\
\hline 1 & 23 & M & Seudocóndilo & 29 & 6 & 23 & 8 & -2 & 22 & 34 & 54 & 37 \\
\hline 2 & 42 & M & No & 34 & 15 & 19 & 19 & -4 & 52 & 59 & 13 & 16 \\
\hline 3 & 20 & $\mathrm{~F}$ & $\begin{array}{l}\text { Inestabilidad } \\
\text { posterolateral }\end{array}$ & 40 & 20 & 20 & 20 & 0 & 46 & 54 & 17 & 12 \\
\hline 4 & 18 & M & No & 22 & 5 & 17 & 6 & -1 & 50 & 61 & 22 & 13 \\
\hline 5 & 34 & M & Seudocóndilo & 25 & 8 & 17 & 11 & -3 & 24 & 37 & 54 & 18 \\
\hline Promedio & 27 & & & 30 & 11 & 19 & 13 & -2 & 39 & 50 & 32 & 19 \\
\hline
\end{tabular}

$\mathrm{M}=$ masculino, $\mathrm{F}=$ femenino

\begin{tabular}{|c|c|c|c|c|c|c|c|c|c|c|c|}
\hline \multirow[t]{2}{*}{ Paciente } & \multicolumn{2}{|c|}{ Flexo-extensión $\left(^{\circ}\right)$} & \multicolumn{2}{|c|}{ MEPS } & \multicolumn{2}{|c|}{ DASH } & \multicolumn{2}{|c|}{ Dolor } & \multirow{2}{*}{$\begin{array}{l}\text { Satisfac- } \\
\text { ción }\end{array}$} & \multirow{2}{*}{$\begin{array}{c}\text { Escala de } \\
\text { Oppenheim }\end{array}$} & \multirow[t]{2}{*}{ Complicaciones } \\
\hline & Preop. & Posop. & Preop. & Posop. & Preop. & Posop. & Preop. & Posop. & & & \\
\hline 1 & $145-0$ & $145-5$ & 65 & 100 & 24 & 2 & 5 & 0 & 9 & Bueno & $\begin{array}{l}\text { Neuritis cubital } \\
\text { y extracción del } \\
\text { implante }\end{array}$ \\
\hline 2 & $135-10$ & $135-10$ & 70 & 100 & 34 & 5 & 5 & 1 & 9 & Excelente & No \\
\hline 3 & $135-0$ & $135-0$ & 65 & 85 & 34 & 13 & 5 & 2 & 9 & Excelente & No \\
\hline 4 & $140-10$ & $140-10$ & 85 & 100 & 9 & 6 & 2 & 1 & 8 & Excelente & No \\
\hline 5 & $135-15$ & $135-5$ & 70 & 100 & 27 & 12 & 3 & 1 & 8 & Excelente & No \\
\hline Promedio & $138-7$ & $138-6$ & 71 & 97 & 26 & 8 & 4 & 1 & 8,6 & & \\
\hline
\end{tabular}

MEPS = Mayo Elbow Performance Score, DASH = Disabilities of the Arm, Shoulder and Elbow .

La evaluación radiográfica preoperatoria arrojó un valgo promedio de $30^{\circ}$ (rango 22-40 ), con un valgo contralateral de $11^{\circ}$ (rango $5-20^{\circ}$ ). La corrección radiográfica mostró un valgo de $13^{\circ}$. Por lo tanto, se obtuvo una corrección, en promedio, de $2^{\circ}$ menos que del lado contralateral. Todas las osteotomías consolidaron y la medición de la prominencia medial fue, en promedio, un 32\% (rango 13-54) mayor que en el preoperatorio (Figuras 2 y 3 ).

Según la escala de Oppenheim, los resultados fueron excelentes ( 4 casos) y bueno ( 1 caso). La satisfacción personal con el resultado fue, en promedio, de 8,6 (rango 8-9). 

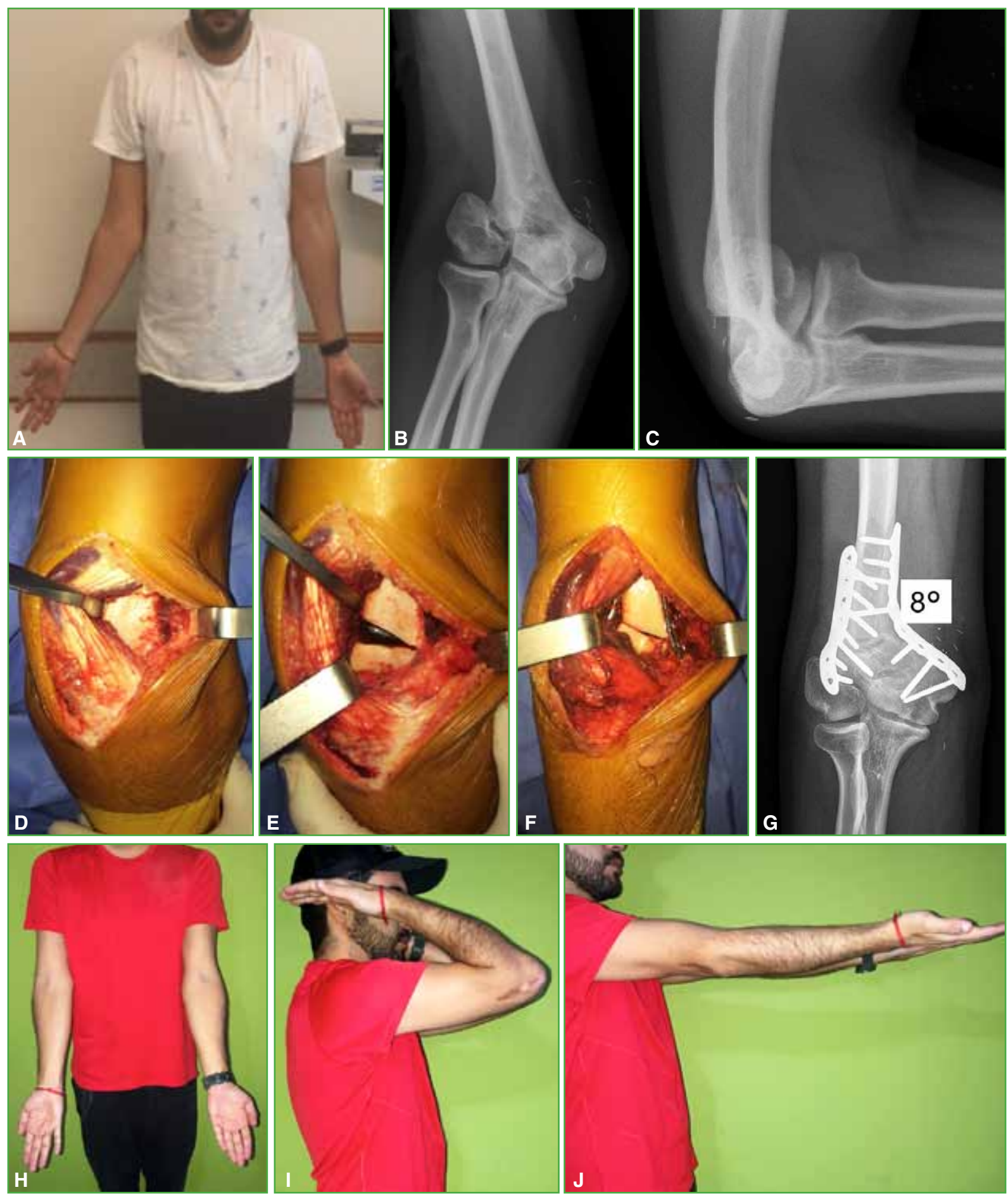

Figura 2. A. Paciente con codo valgo (Caso 1). B y C. Radiografía de frente y de perfil de codo, con seudoartrosis del cóndilo humeral y desviación en valgo de $29^{\circ}$. D. Abordaje paratricipital. E. Osteotomía en cuña sustractiva. F. Osteosíntesis de la osteotomía. G. Radiografía con valgo posoperatorio de $8^{\circ}$. H-J. Movilidad final. 

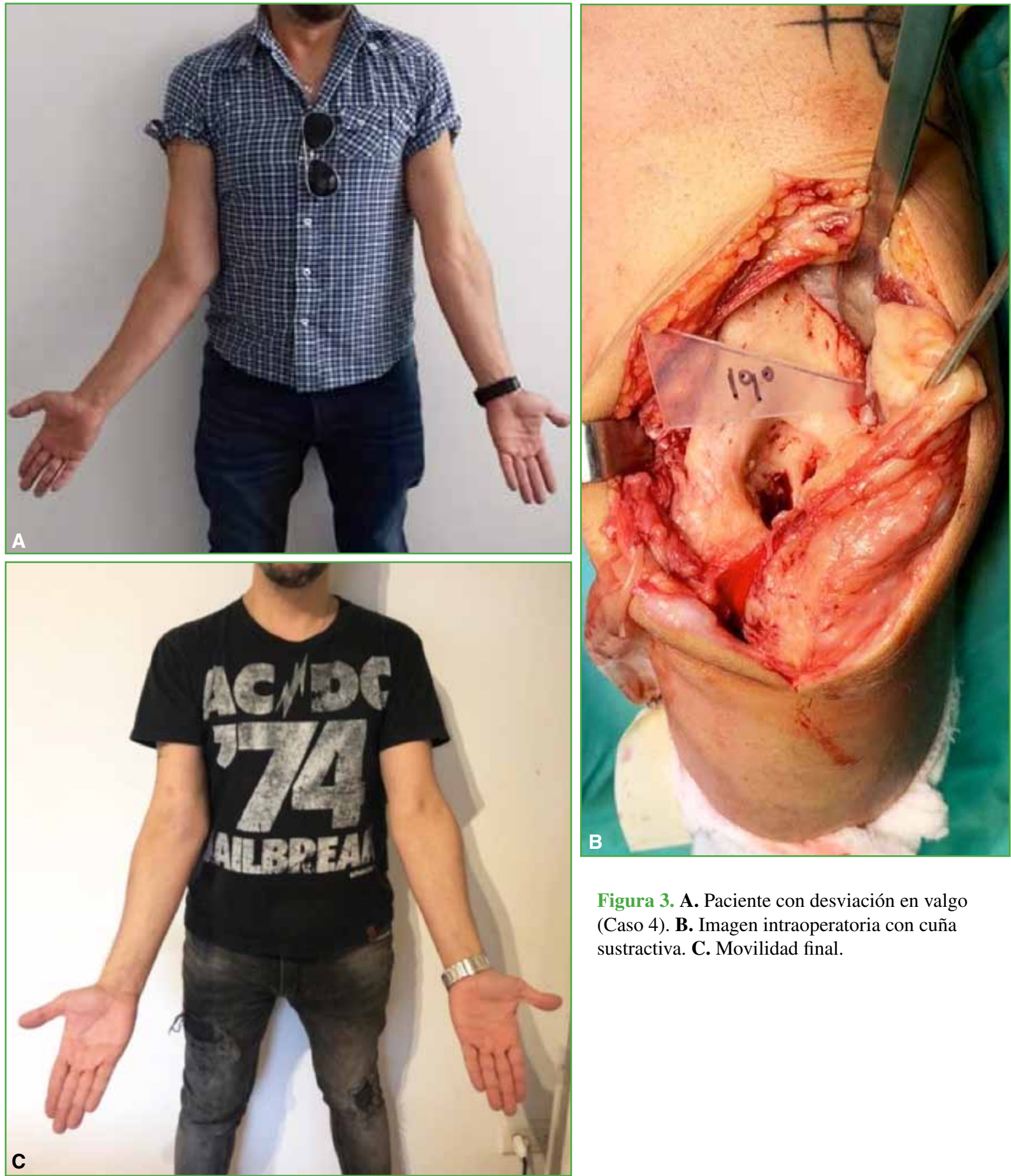

Figura 3. A. Paciente con desviación en valgo (Caso 4). B. Imagen intraoperatoria con cuña sustractiva. C. Movilidad final.

\section{DISCUSIÓN}

Comunicamos los resultados de la osteotomía en cuña monoplanar para el tratamiento del codo valgo y hemos obtenido buenos resultados funcionales. Este tipo de osteotomía es ampliamente criticada en la bibliografía, porque la deformidad, a menudo, es multiplanar, por lo que, para muchos autores, así debe ser su corrección. La osteotomía que presentamos es monoplanar, y no permitiría la corrección en todos los planos. ${ }^{6,714,15}$ Las críticas a esta osteotomía incluyen: que no permite una corrección rotatoria, provee menor estabilidad por ser una osteotomía lineal y aumenta la prominencia medial, pues no permite la traslación del fragmento distal. 
Las tres osteotomías publicadas con más frecuencia son la de cuña en cierre, la traslacional escalonada en V y la cupuliforme. La osteotomía traslacional escalonada tiene algunas ventajas, como proporcionar una estabilidad intrínseca a partir de la forma en V de los cortes y permitir una corrección multiplanar. Kin y cols. ${ }^{8}$ publicaron los resultados de 13 pacientes con esta técnica. Lograron una buena corrección angular, con un valgo final promedio de $9,1^{\circ}$ y una mejoría de la prominencia medial. Sin embargo, cuatro pacientes necesitaron de una osteotomía adicional para corregir una contractura en flexión.

La osteotomía cupuliforme o en domo es menos compleja que la anterior, pero la corrección, a nuestro criterio, es menos precisa que con la resección de una cuña. Hahn y cols. ${ }^{8}$ reportaron 13 pacientes sometidos a esta osteotomía, con una corrección del valgo de $24^{\circ}$ a $11^{\circ}$, con mejoría de los puntajes clínico-funcionales y de la prominencia medial.

Si bien estos tipos de osteotomías tendrían la ventaja de la corrección multiplanar, no hay estudios publicados que comparen los resultados con las osteotomías monoplanares. En nuestra serie, el aumento de la prominencia medial fue del $32 \%$. Si bien esta es una de las críticas a esta técnica, la satisfacción clínica de los pacientes ha sido alta y ninguno refirió molestias con respecto a este punto.

Por lo tanto, consideramos que, salvo casos excepcionales, como ante una deformidad muy marcada en otros planos, además del coronal, la corrección monoplanar permite lograr similares resultados a los de otras técnicas multiplanares y con una técnica más sencilla.

No se trató la seudoartrosis del cóndilo humeral, porque no eran sintomáticas. Si bien algunos autores recomiendan la osteosíntesis de la seudoartrosis, ${ }^{14,16}$ otros han obtenido buenos resultados sin tratamiento quirúrgico. ${ }^{17}$

Este estudio presenta muchas limitaciones. En primer lugar, su carácter retrospectivo. En segundo lugar, la serie estaba formada por una escasa cantidad de pacientes con un seguimiento acotado. En tercer lugar, la evaluación funcional final estuvo a cargo del cirujano a cargo. Sin embargo, este tipo de deformidad es un cuadro poco comunicado en nuestra bibliografía y reivindica este tipo de osteotomía muchas veces criticada.

La osteotomía varizante en cuña sustractiva para el tratamiento de un codo valgo del adulto es una buena opción terapéutica, se recuperan los valores angulares comparables con los del lado contralateral y la tasa de satisfacción de los pacientes es alta. Al ser una técnica menos compleja que las osteotomías multiplanares, surge como nuestra elección ante una consolidación viciosa en valgo del codo.

Conflicto de intereses: Los autores no declaran conflictos de intereses.

ORCID de G. Altube: https://orcid. org/0000-0001-6113-449X

ORCID de J. G. Boretto: https://orcid.org/0000-0001-7701-3852

ORCID de A. Donndorff: hitps:///orcid.org/0000-0002-6384-4820
ORCID de E. E. Zaidenberg: https://orcid org/0000-0002-1535-0586

ORCID de I. Rellán: https://orcid.org/0000-0003-4045-339X

ORCID de P. De Carli: https://orcid.org/0000-0002-9474-8129

\section{BIBLIOGRAFÍA}

1. Arnold JA, Nasca RH, Nelson CL. Supracondylar fracture of the humerus: The role of dynamic factors in prevention of deformity. J Bone Joint Surg Am 1977;59:589-95. PMID: 873954

2. Storm SW, Williams DP, Khoury J, Lubahn JD. Elbow deformities after fracture. Hand Clin 2006;22(1):121-9. https://doi.org/10.1016/j.hcl.2005.12.005

3. Bub FR, Schulz AP, Lill H, Voigt C. Supracondylar osteotomies of posttraumatic distal humeral deformities in young adults-technique and results. Open Orthop J 2011;5:389-94. https://doi.org/10.2174/1874325001105010389

4. Labelle H, Bunnell WP, Duhaime M, Poitras B. Cubitus varus deformity following supracondylar fractures of the humerus in children. J Pediatr Orthop 1982;2(5):539-46. https://doi.org/10.1097/01241398-198212000-00014

5. Wilkins KE. Fractures and dislocations of the elbow region. En: Rockwood CA, Wilkins KE, King RE (eds.). Fractures in children, $4^{\text {th }}$ ed. Philadelphia: Lippincott-Raven; 1996:600-887. 
6. Amspacher JC, Messenbaugh JF Jr. Supracondylar osteotomy of the humerus for correction of rotational and angular deformities of the elbow. South Med J 1964; 57:846-50. https://doi.org/10.1097/00007611-196407000-00022

7. Kang HJ, Koh IH, Jeong YC, Yoon TH, Choi YR. Efficacy of combined osteotomy and ulnar nerve transposition for cubitus valgus with ulnar nerve palsy in adults. Clin Orthop Relat Res 2013;471(10):3244-50. https://doi.org/10.1007/s11999-013-3057-9

8. Kim HT, Lee JS, Yoo CI. Management of cubitus varus and valgus. J Bone Joint Surg Am 2005;87:771-80. https://doi.org/10.2106/JBJS.D.01870

9. Wong HK, Lee EH, Balasubramaniam P. The lateral condylar prominence. A complication of supracondylar osteotomy for cubitus varus. J Bone Joint Surg Br 1990;72:859-61. PMID: 2211772

10. Milch H. Fractures and fracture dislocations of the humeral condyles. J Trauma 1964;4:592-607. https://doi.org/10.1097/00005373-196409000-00004

11. Hudak PL, Amadio PC, Bombardier C. Development of an upper extremity outcome measure: the DASH (Disabilities of the Arm, Shoulder and Hand) [corrected]. The Upper Extremity Collaborative Group (UECG). Am J Ind Med 1996;29:602-8. https://doi.org/10.1002/(SICI)1097-0274(199606)29:6<602::AID-AJIM4>3.0.CO;2-L

12. Morrey BF, An KN, Chao EYS. Functional evaluation of the elbow. En: Morrey BF (ed.). The elbow and its disorders. $2^{\text {nd }}$ ed. Philadelphia, PA: WB Saunders Co.; 1993:95.

13. Oppenheim WL, Clader TJ, Smith C, Bayer M. Supracondylar humeral osteotomy for traumatic childhood cubitus varus deformity. Clin Orthop Relat Res 1984;188:34-9. PMID: 6467726

14. Abed Y, Nour K, Kandil YR, El-Negery A. Triple management of cubitus valgus deformity complicating neglected nonunion of fractures of lateral humeral condyle in children: a case series. Int Orthop 2018;42(2):375-84. https://doi.org/10.1007/s00264-017-3709-6

15. Tachdjian MR. Osteotomy of distal humerus for correction of cubitus varus. En: Smith AB (ed.). Pediatric orthopedics. Philadelphia: WB Saunders; 1972:1588-91.

16. Roye DP Jr, Bini SA, Infosino A. Late surgical treatment of lateral condylar fractures in children. J Pediatr Orthop 1991;11:195-9. https://doi.org/10.1097/01241398-199103000-00011

17. Fontanetta P, Mackenzie DA, Rosman M. Missed, maluniting, and malunited fractures of the lateral humeral condyle in children. J Trauma 1978;18:329-35. https://doi.org/10.1097/00005373-197805000-00006 